\title{
Protective effect of hydrogen-rich water on liver function of colorectal cancer patients treated with mFOLFOX6 chemotherapy
}

\author{
QINGXI YANG $^{1}$, GUANGDONG JI $^{1}$, RONGTAO PAN ${ }^{1}$, YINGHUI ZHAO ${ }^{2}$ and PENG YAN ${ }^{3}$ \\ ${ }^{1}$ Department of Oncology, Shandong Provincial Taishan Hospital; ${ }^{2}$ Department of Pathology, \\ Taishan Medical University, Taian, Shandong 271000; ${ }^{3}$ Department of Oncology, Jinan Central \\ Hospital Affiliated to Shandong University, Jinan, Shandong 250013, P.R. China
}

Received January 31, 2017; Accepted June 23, 2017

DOI: $10.3892 /$ mco.2017.1409

\begin{abstract}
The present study was conducted to investigate the protective effect of hydrogen-rich water on the liver function of colorectal cancer (CRC) patients treated with mFOLFOX6 chemotherapy. A controlled, randomized, single-blind clinical trial was designed. A total of 152 patients with CRC were recruited by the Department of Oncology of Taishan Hospital (Taian, China) between June 2010 and February 2016, among whom 146 met the inclusion criteria. Subsequently, 144 patients were randomized into the treatment $(n=80)$ and placebo $(n=64)$ groups. At the end of the study, 76 patients in the hydrogen treatment group and 60 patients in the placebo group were included in the final analysis. The changes in liver function after the chemotherapy, such as altered levels of alanine aminotransferase (ALT), aspartate transaminase (AST), alkaline phosphatase, indirect bilirubin (IBIL) and direct bilirubin, were observed. The damaging effects of the mFOLFOX6 chemotherapy on liver function were mainly represented by increased ALT, AST and IBIL levels. The hydrogen-rich water group exhibited no significant differences in liver function before and after treatment, whereas the placebo group exhibited significantly elevated levels of ALT, AST and IBIL. Thus, hydrogen-rich water appeared to alleviate the mFOLFOX6-related liver injury.
\end{abstract}

\section{Introduction}

Antitumor agents are mostly cytotoxic drugs, which inevitably lead to damage of normal tissue cells and organs, or cause adverse reaction when killing cancer cells. Chemotherapeutic agents may cause liver damage, mainly including necrosis or fatty degeneration of liver cells, cholestasis and liver vessel damage (1-4). Hepatic dysfunction tends to affect the course

Correspondence to: Dr Peng Yan, Department of Oncology, Jinan Central Hospital Affiliated to Shandong University, 105 Jie Fang Road, Jinan, Shandong 250013, P.R. China

E-mail: phoenix142@126.com

Key words: colorectal cancer, hydrogen-rich water, chemotherapy, mFOLFOX6, liver damage of antitumor treatment, increasing patient discomfort and the overall financial burden.

Hydrogen is a naturally existing colorless, tasteless and odorless gas, and its protective effect against oxidative damage to the brain, liver, kidney and other major organs has been previously described (5). Solubilized hydrogen (hydrogen-rich water) is a portable, easily administered and safe means of delivering molecular hydrogen (6). The aim of the present prospective study was to administer hydrogen-rich water to colorectal cancer (CRC) patients treated with mFOLFOX6 chemotherapy by a randomized, single-blind, controlled clinical research method, and compare the chemotherapy-induced liver damage between the treatment and control groups.

\section{Subjects and methods}

Subjects. A total of 152 patients with CRC were recruited at the Department of Oncology of Taishan Hospital (Taian, China) between June 2010 and February 2016, among whom 146 met the inclusion criteria. Subsequently, 144 patients were randomized into the treatment $(n=80)$ and placebo $(n=64)$ groups. At the end of the study, 76 patients in the hydrogen treatment group and 60 patients in the placebo group were included in the final analysis (Fig. 1).

The 136 subjects were aged 41-86 years (mean age, $55.6 \pm 14.2$ years) and included 56 men and 80 women and the number of elderly subjects (aged $\geq 70$ years) was 78 .

Inclusion criteria. Stage $\geq$ IIB CRC (after surgery or inoperable); need for mFOLFOX6 chemotherapy; age >18 years; Eastern Cooperative Oncology Group (ECOG) performance status (PS) score $<1$; all cases had a definitive pathological diagnosis.

Exclusion criteria. Viral hepatitis, carriers of viral hepatitis, alcoholic hepatitis, drug-induced hepatitis and autoimmune hepatitis, hematological diseases, heart diseases, hyperthyroidism, pregnancy, hepatic cirrhosis and neuropsychiatric disorders. This was a controlled, randomized, single-blind study. The study protocol was approved by the Ethics Committee of Shandong Provincial Taishan Hospital, and written informed consent was obtained from all the subjects prior to enrollment. 


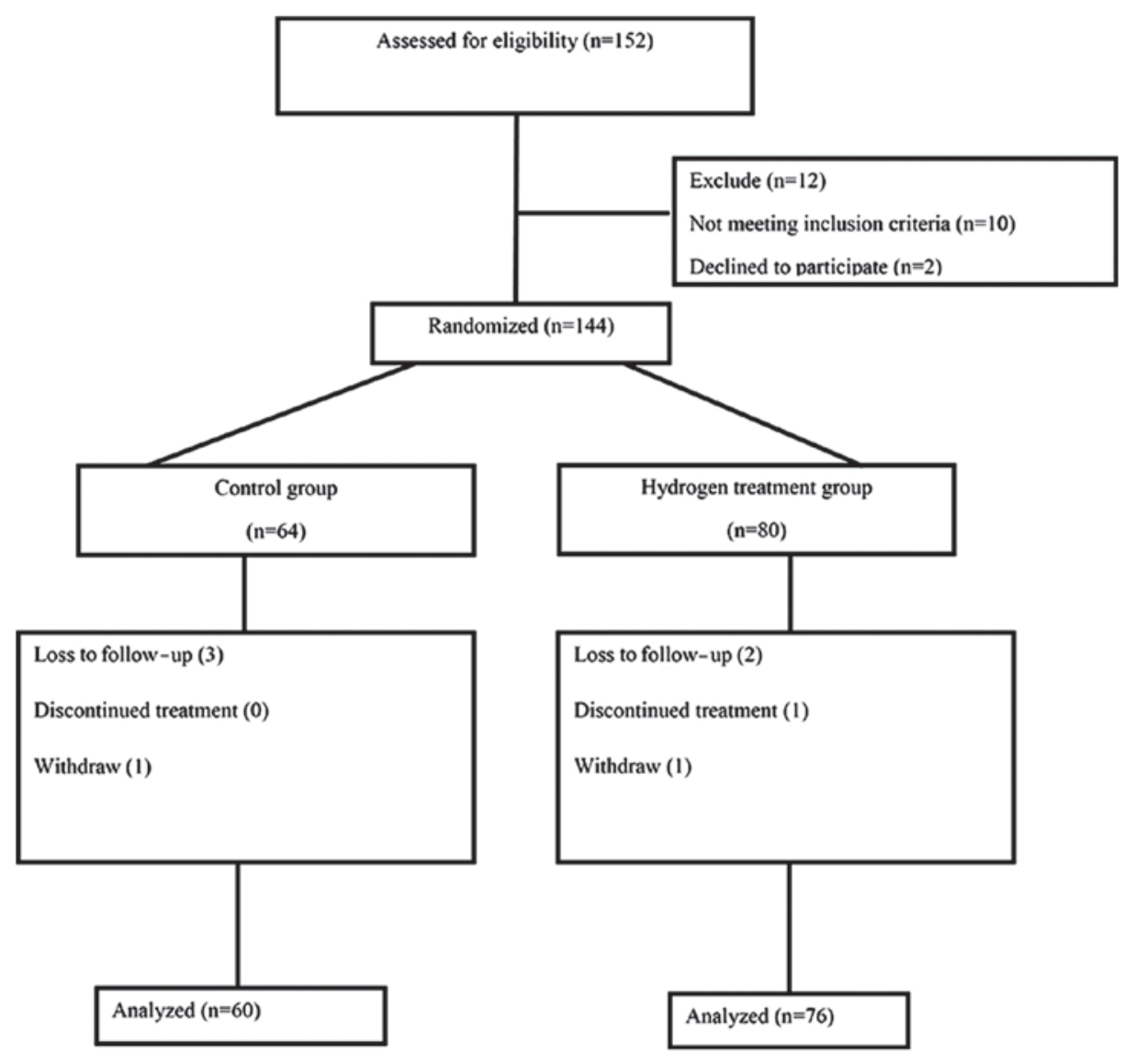

Figure 1. Flow chart of patient recruitment process.

Preparation of hydrogen-rich water. Hydrogen-rich water was prepared by increasing the hydrogen pressure in the solution (7). First, the partial air pressure in the water was reduced using a 1406 type vacuum pump (Shanghai Medical Equipment Works Co., Ltd., Shanghai, China). The solution was then passed through hydrogen cylinders with a $99.999 \%$ hydrogen purity for $2 \mathrm{~h}$ to obtain a water solution rich in hydrogen. The amount of dissolved hydrogen in hydrogen-rich water was measured using an ENH-1000 portable meter (TRUSTLEX Co., Ltd., Tokyo, Japan), as this water is only considered drinkable under the level of 0.27-0.4 ppm.

Chemotherapy regimen and randomization. All the subjects received mFOLFOX6 chemotherapy. Dosage: Oxaliplatin $85 \mathrm{mg} / \mathrm{m}^{2}$ on day 1 as an i.v. drip, calcium folinate $400 \mathrm{mg} / \mathrm{m}^{2}$ on day 1 i.v., 5-fluorouracil (5-FU) 2,400 mg/m² as a continuous i.v. infusion over 46 h (National Comprehensive Cancer Network guidelines 2012, version 1) (8). The baseline characteristics of patients in the two groups are summarized in Table I.

Intake of hydrogen-rich water. The patients in the hydrogen-rich water group started drinking hydrogen-rich water 1 day prior to chemotherapy until the end of the cycle, for a total of 4 days, with a daily intake of 1,000 $\mathrm{ml}$ in 4 doses (250 ml each). Hydrogen-rich water was consumed $0.5 \mathrm{~h}$ after a meal and before bedtime. The patients did not discontinue consuming hydrogen-rich water during the entire course of chemotherapy. The patients in the placebo group consumed distilled water, with a daily intake of $1,000 \mathrm{ml}$ in 4 doses (250 $\mathrm{ml}$ each).

Assessment of liver function. Alanine aminotransferase (ALT), aspartate transaminase (AST), alkaline phosphatase (ALP), direct bilirubin (DBIL) and indirect bilirubin (IBIL) were measured with an automatic biochemical analyzer (TBA-120R; Toshiba, Tokyo, Japan), and the reagents (no. 12125) were purchased from Whiteman Biotech Co., Ltd. (Nanjing, China). The measurements were performed according to the manufacturer's instructions. Liver function assessment was performed on the 10th day after chemotherapy. The standard classification of liver toxicity following chemotherapy was shown in Table II (WHO standards).

Statistical analysis. All data are presented as mean \pm standard deviation. Measurement data were analyzed using independent-samples t-test and numerical data with the use of the rank-sum test. All data analyses were performed with SPSS software, version 13.0 (SPSS Inc., Chicago, IL, USA). $\mathrm{P}<0.05$ was considered to indicate statistically significant differences.

\section{Results}

Patients. As shown in Table I, a total of 136 patients were analyzed. There was no statistically significant difference in the stratification of all factors (age, gender, ECOG PS, smoking history, history of alcohol consumption, hepatic metastases, chemotherapy cycles) between the two groups 
Table I. Baseline characteristics of patients.

\begin{tabular}{|c|c|c|c|c|}
\hline \multirow[b]{2}{*}{ Characteristics } & \multirow[b]{2}{*}{ Total } & \multicolumn{2}{|c|}{ Groups } & \multirow[b]{2}{*}{ P-value } \\
\hline & & Hydrogen-rich water $(n=76)$ & Control $(n=60)$ & \\
\hline Age (years) & & & & 0.919 \\
\hline$<70$ & 58 & 32 & 26 & \\
\hline$\geq 70$ & 78 & 44 & 34 & \\
\hline Gender & & & & 0.243 \\
\hline Male & 56 & 36 & 20 & \\
\hline Female & 80 & 40 & 40 & \\
\hline ECOG PS score & & & & 0.340 \\
\hline $0-1$ & 82 & 42 & 40 & \\
\hline 2 & 54 & 34 & 20 & \\
\hline Smoking history & & & & 0.174 \\
\hline Yes & 76 & 48 & 28 & \\
\hline No & 60 & 28 & 32 & \\
\hline History of alcohol intake & & & & 0.457 \\
\hline Yes & 38 & 18 & 20 & \\
\hline No & 94 & 54 & 40 & \\
\hline History of hepatitis & & & & 0.660 \\
\hline Yes & 46 & 24 & 22 & \\
\hline No & 90 & 52 & 38 & \\
\hline Hepatic metastases & & & & 0.382 \\
\hline Yes & 48 & 24 & 24 & \\
\hline No & 72 & 36 & 36 & \\
\hline No. of chemotherapy cycles & & & & 0.265 \\
\hline $1-3$ & 80 & 40 & 40 & \\
\hline $3-6$ & 40 & 22 & 18 & \\
\hline$>6$ & 16 & 10 & 6 & \\
\hline
\end{tabular}

Statistical significance was set at $\mathrm{P}<0.05$. ECOG PS, Eastern Cooperative Oncology Group performance status.

Table II. Standard classification of liver toxicity following chemotherapy according to the World Health Organization guidelines.

\begin{tabular}{llcccc}
\hline Classification & \multicolumn{1}{c}{ Grade 0} & Grade 1 & Grade 2 & Grade 3 & Grade 4 \\
\hline BIL & $\mathrm{N}=1.71-17.1 \mu \mathrm{mol} / \mathrm{l}$ & $<1.5 * \mathrm{~N}$ & $(1.5-3) * \mathrm{~N}$ & $(3-10) * \mathrm{~N}$ & $>10 * \mathrm{~N}$ \\
AST/ALT & $\mathrm{N}=0-40 \mathrm{IU} / \mathrm{l}$ & $\leq 2.5 * \mathrm{~N}$ & $(2.6-5) * \mathrm{~N}$ & $(5.1-20) * \mathrm{~N}$ & $>20 * \mathrm{~N}$ \\
ALP & $\mathrm{N}=25-90 \mathrm{IU} / \mathrm{l}$ & & & Pre-hepatic coma & Hepatic coma \\
Hepatic coma & No changes before/after treatment & - & - & & \\
\hline
\end{tabular}

$\mathrm{N}$, upper limit of normal value; ALT, alanine aminotransferase; AST, aspartate transaminase; ALP, alkaline phosphatase; DBIL, direct bilirubin; IBIL, indirect bilirubin.

$(\mathrm{P}>0.05)$; thus, randomization was considered as baseline balance.

Effect of mFOLFOX6 on liver function. As shown in Table III, the effect of mFOLFOX6 chemotherapy on the liver was mainly characterized by elevated ALT, AST and IBIL levels. The number of cases with abnormal ALT, AST, ALP, DBIL and IBIL levels following chemotherapy was 46, 40,11, 6 and 17, respectively, accounting for $33.82,29.41,8.09,4.41$ and $12.50 \%$, respectively.
Comparison of liver damage between hydrogen-rich water and placebo groups. The probability and degree of chemotherapy-induced liver damage in the hydrogen-rich water group were lower compared with those in the placebo group. The comparison of hepatic injury following chemotherapy between the two groups was performed with the use of the rank-sum test. As shown in Table IV, the patients in the hydrogen-rich water group had a lower probability and degree of hepatic damage compared with those in the placebo group $(\mathrm{P}<0.05)$. 
Table III. Types of hepatic damage in patients exhibiting mFOLFOX6-induced liver injury.

Abnormal marker, patient no. (\%)

\begin{tabular}{lcccc}
\hline AST & ALT & ALP & DBIL & IBIL \\
\hline $46(33.82)$ & $40(29.41)$ & $11(8.09)$ & $6(4.41)$ & $17(12.50)$ \\
\hline
\end{tabular}

mFOLFOX6, 5-FU, oxaliplatin and calcium folinate; ALT, alanine aminotransferase; AST, aspartate transaminase; ALP, alkaline phosphatase; DBIL, direct bilirubin; IBIL, indirect bilirubin.

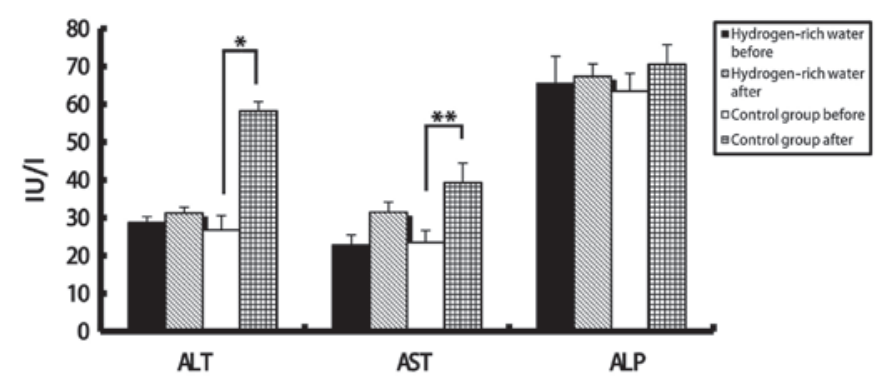

Figure 2. Comparison of changes in ALT, AST and ALP levels before and after chemotherapy between the two groups. ALT, alanine aminotransferase; AST, aspartate aminotransferase; ALP, alkaline phosphatase. " $\mathrm{P}=0.04$; *** $\mathrm{P}=0.032$.

Comparison of liver function tests before and after treatment between the two groups. There were no statistically significant differences in the levels of ALT, AST, ALP, DBIL and IBIL in the hydrogen-rich water group before and after chemotherapy. However, the difference in ALT, AST and DBIL levels before and after chemotherapy in the control group was statistically significant (seen in Figs. 2 and 3). The ALT levels in the hydrogen-rich water group before and after chemotherapy were $28.72 \pm 1.6$ and $31.28 \pm 1.47 \mathrm{IU} / 1$, respectively; the difference was not statistically significant $(\mathrm{P}=0.46)$. The ALT levels in the control group before and after chemotherapy were $26.78 \pm 3.8$ and $58.22 \pm 2.46 \mathrm{IU} / 1$, respectively; the difference was statistically significant $(\mathrm{P}=0.04)$. The AST levels in the hydrogen-rich water group before and after chemotherapy were $22.74 \pm 2.74$ and $23.43 \pm 2.66 \mathrm{IU} / 1$, respectively; the difference was not statistically significant $(\mathrm{P}=0.67)$. The AST in the control group before and after chemotherapy were $23.43 \pm 3.24$ and $39.28 \pm 5.17 \mathrm{IU} / 1$, respectively; the difference was statistically significant $(\mathrm{P}=0.032)$. The ALP levels in the hydrogen-rich water group before and after chemotherapy were $65.52 \pm 7.13$ and $67.34 \pm 3.32 \mathrm{IU} / 1$, respectively; the difference was not statistically significant $(\mathrm{P}=0.45)$. The ALP levels in the control group before and after chemotherapy were $63.44 \pm 4.70$ and $70.52 \pm 5.22 \mathrm{IU} / 1$, respectively; the difference was not statistically significant $(\mathrm{P}=0.70)$. The DBIL levels in the hydrogen-rich water group before and after chemotherapy were $2.2 \pm 0.07$ and $3.48 \pm 0.10 \mu \mathrm{mol} / 1$, respectively; the difference was not statistically significant $(P=0.44)$. The DBIL levels in the control group before and after chemotherapy were $2.42 \pm 0.04$ and $3.34 \pm 0.05 \mu \mathrm{mol} / 1$ respectively; the difference was not statistically significant $(\mathrm{P}=0.32)$. The IBIL levels in the hydrogen-rich water group before and after chemotherapy were $11.70 \pm 1.02$ and $14.20 \pm 1.44 \mu \mathrm{mol} / 1$, respectively; the difference was not statistically significant $(\mathrm{P}=0.10)$. The IBIL levels in the control group before and after chemotherapy were $10.98 \pm 3.17$ and $20.70 \pm 3.07 \mu \mathrm{mol} / 1$, respectively; the difference was statistically significant $(\mathrm{P}=0.046)$.

\section{Discussion}

Chemotherapeutic agents and their metabolites may directly induce damage to hepatic parenchymal cells; in addition, they may also cause further damage to the hepatocytes through cellular or humoral immunity mechanisms. There are two major categories of hepatic tissue damage commonly occurring as a consequence of chemotherapy: One is similar to non-alcoholic fatty liver disease and is often referred to as chemotherapy-associated steatohepatitis (CASH) (9); the other results from injury to the sinusoids causing venous congestion. Endothelial cells in the sinusoids become damaged, leading to initiation of the coagulation cascade within the subendothelial space of Disse and, ultimately, to sinusoidal obstruction, as fibrotic changes occur in the central venules (10).

Of the 136 patients included in the present study, 58 (42.6\%) experienced post-chemotherapeutic hepatic function compromise. In the majority of the cases (75.9\%), the liver injury was grade $1-2$. The mechanism underlying chemotherapeutic agent-induced liver injury is as follows: The majority of chemotherapeutic agents are metabolized in the liver; when the chemotherapeutic agent and its metabolites are beyond the hepatic metabolic abilities, the electrophilic products and superoxide ions generated via the metabolic process damage the hepatocellular, hepatic mitochondrial and microsomal membranes, directly inducing hepatocellular injury through covalent bonding and promotion of lipid peroxidation; in addition, the drug metabolites form oxygen-free radicals promoting lipid peroxidation, indirectly inducing hepatocellular injury.

mFOLFOX6 chemotherapy consists of 5-FU, oxaliplatin and calcium folinate, once every 2 weeks, which is associated with high risk of hepatotoxicity. 5 -FU is converted in vivo into a triple complex, which is difficult to depolymerize, including fluorodeoxyuridine phosphate, thymidylate synthase and 5,10-citrovorum factor; it inhibits thymidylate synthase and also blocks the synthesis of deoxythymidylic acid, eventually affecting the synthesis of deoxynucleotides. Oxaliplatin may act on DNA through the generation of alkylation conjugates, forming intrachain and interstrand cross-links, and generating cytotoxic effects. 5-FU is more associated with steatosis (11), whereas oxaliplatin regimens may result in sinusoidal injuries leading to sinusoidal obstruction syndrome $(12,13)$. 5-FU specifically is considered to affect mitochondrial membranes, allowing for an increase in reactive oxygen species and setting off a cascade of events leading to lipid peroxidation, fibrosis and cell death $(14,15)$. Sinusoidal injury is also considered to result from reactive oxygen species. Once endothelial cells were injured, the coagulation cascade is activated and may lead to sinusoidal obstruction (16).

A comparison of several indicators of the liver function was performed; the post-chemotherapeutic liver injuries mainly manifested as elevation in ALT, AST and IBIL levels, with 
Table IV. Comparison of hepatic damage between the hydrogen-rich water and control groups.

\begin{tabular}{lccccrr}
\hline Groups & Grade 0 & Grade 1 & Grade 2 & Grade 3 & Grade 4 & Mean-rank \\
\hline Hydrogen-rich water & 54 & 14 & 4 & 4 & 0 & 58.13 \\
Control & 24 & 16 & 10 & 8 & 2 & 81.63 \\
P-value & & & & & 0.00 \\
\hline
\end{tabular}

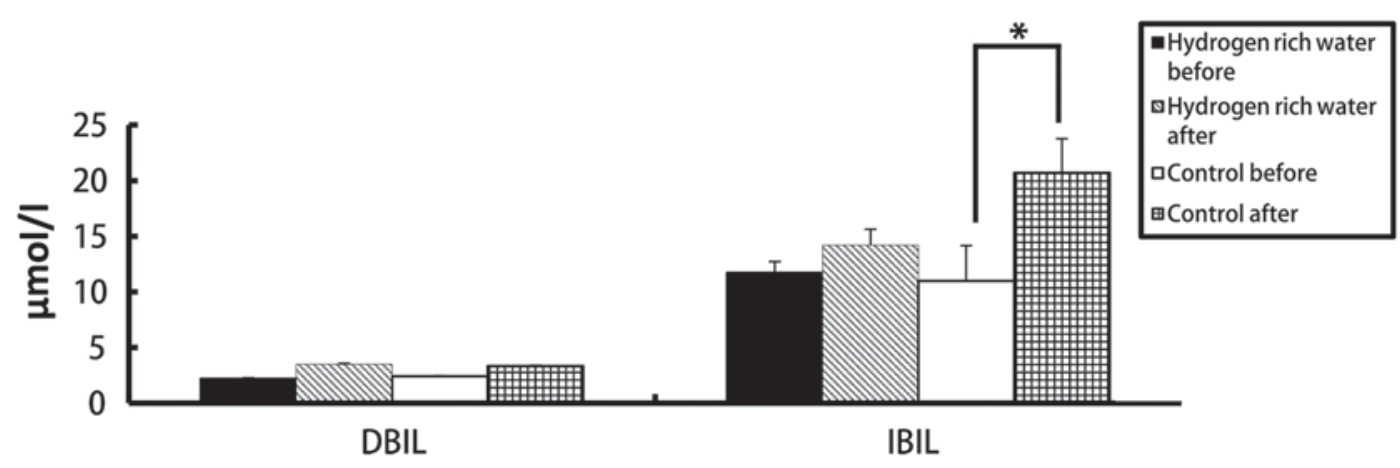

Figure 3. Comparison of changes in DBIL and IBIL levels before and after chemotherapy between the two groups. ${ }^{*} \mathrm{P}=0.046$.

46 cases with ALT abnormalities, 40 cases of AST abnormalities, and 17 cases of elevation in IBIL levels. Considering that the patients received mFOLFOX6 chemotherapy, post-chemotherapeutic liver injury mainly manifested as elevation in ALT, AST and IBIL levels.

Chemotherapeutic agents injure hepatocytes, mainly through interfering with hepatocellular metabolism and forming oxygen free radicals, inducing hepatocellular necrosis and inflammation, or the damages are induced by hepatic fibrosis, fatty degeneration and sinusoidal obstruction. ALT and AST, which are mainly distributed in the hepatocellular cytoplasm, are the most sensitive indicators reflecting hepatocellular inflammatory injury. ALT and AST are released from damaged cells into the blood. Therefore, chemotherapy leads to an elevation in liver enzyme and IBIL levels. ALP is elevated when the bile excretion is blocked with hepatocellular injuries. TBIL is generated by the liver and excreted through the biliary tract; an elevation in serum DBIL indicates inhibition of bile excretion, or a disorder in liver uptake and bilirubin secretion. Chemotherapy does not lead to biliary tract inflammation or obstruction, nor to elevation in serum DBIL and ALP levels. However, Nakano et al reported that gemcitabine may lead to bile duct obstruction and cholestasis (17).

Some studies suggest that $>6$ cycles of chemotherapy is an independent prognostic factor of liver injury (17). In addition, patients with elevated body mass index, type 2 diabetes mellitus, or metabolic syndrome, have an increased risk of steatosis, irrespective of chemotherapy (18).

In 2007, Ohsawa et al (19) observed that molecular hydrogen selectively reduced cytotoxic reactive oxygen species in vitro and exerted a therapeutic antioxidant effect. It has been demonstrated that $\mathrm{H}_{2}$ exerted preventive or therapeutic effects on cerebral, myocardial and hepatic ischemia-reperfusion injuries, intestinal, lung, renal and heart transplantation, and acute graft-versus-host disease post-allogeneic hematopoietic stem cell transplantation (20,21). Recent basic and clinical research revealed that hydrogen is an important physiological regulatory factor with antioxidant, anti-inflammatory and anti-apoptotic protective effects on cells and organs (22-25).

Using hydrogen as a potential antioxidant has multiple advantages: It may effectively neutralize hydroxy radicals present in live cells, unlike most known antioxidants, which cannot successfully enter the target organelles. Hydrogen has good distribution characteristics, and it can penetrate biomembranes and diffuse into the cytoplasm, mitochondrion and nucleus. Although hydrogen's activity is mild, its rapid gas diffusion properties are very effective in reducing the toxicity of free radicals in cells.

Previous studies have indicated that inhalation of $1 \%$ hydrogen gas or oral administration of hydrogen-rich water may reduce organ toxicity induced by cisplatin chemotherapy, improve the quality of life and decelerate weight loss, without affecting the effectiveness of chemotherapy (26). As reported, hydrogen-rich water may reduce hepatic injury through reducing oxidative stress and high mobility group box 1 (27). In vitro experimental studies have indicated that hydrogen-rich water may reduce the liver damage induced by obstructive jaundice and endotoxins $(28,29)$.

In the present study, patients in chemotherapy who were treated with hydrogen had no significant differences in liver function indicators, such as ALT, AST, ALP, IBIL or DBIL after chemotherapy, indicating that hydrogen does exert a protective effect on liver function.

Hydrogen-rich water is an easily-accessible, safe, cost-effective and promising treatment. The hydrogen gas mainly enters the blood circulation following entry into the human body and is mainly excreted with respiration, it is not metabolized via the liver or kidneys, and it has no toxicity on human body (30). 
It is unknown whether hydrogen-rich water exerts anti-oxidative stress effects on tumor tissues, and the local control rate, progression-free survival and overall survival must be observed through patient follow-up to verify that hydrogen-rich water does not compromise the effectiveness of chemotherapy.

In the present study, hydrogen-rich water exhibited good efficacy and safety in protecting liver function. However, a large number of randomized clinical trials are required to confirm whether this treatment may be applied in the clinical setting and whether it affects the efficacy of chemotherapy.

\section{Acknowledgements}

The authors would like to thank the Department of Pathology, Taishan Medical University. The present study was supported by the Taian Science and Technology Fund (grant no. 2015NS2098).

\section{References}

1. Christova TY, Gorneva GA, Taxirov SI, Duridanova DB and Setchenska MS: Effect of cisplatin and cobalt chloride on antioxidant enzymes in the livers of Lewis lung carcinoma bearing mice: Protective role of heme oxygenase. Toxicol Lett 138 235-242, 2003.

2. Koc A, Duru M, Ciralik H, Akcan R and Sogut S: Protective agent, erdosteine, against cisplatin-induced hepatic oxidant injury in rats. Mol Cell Biochem 278: 79-84, 2005.

3. Robinson K, Lambiase L, Li J, Monteiro C and Schiff M: Fatal cholestatic liver failure associated with gemcitabine therapy. Dig Dis Sci 48: 1804-1808, 2003.

4. Bibeau F, Azria D, Chateau MC, Borrelly C, Ychou M, Quenet F and Rouanet P: Vascular hepatic injury following neoadjuvant treatment for a cardial adenocarcinoma. Gastroenterol Clin Biol 30: 611-613, 2006 (In French).

5. Huang CS, Kawamura T, Toyoda Y and Nakao A: Recent advances in hydrogen research as a therapeutic medical gas. Free Radic Res 44: 971-282, 2010.

6. Cardinal JS, Zhan J, Wang Y, Sugimoto R, Tsung A, McCurry KR, Billiar TR and Nakao A: Oral hydrogen water prevents chronic allograft nephropathy in rats. Kidney Int 77: 101-109, 2010

7. Mao YF, Zheng XF, Cai JM, You XM, Deng XM, Zhang JH, Jiang L and Sun XJ: Hydrogen-rich saline reduces lung injury induced by intestinal ischemia/reperfusion in rats. Biochem Biophys Res Commun 381: 602-605, 2009.

8. National Comprehensive Cancer Network: (NCCN) Clinical Practice Guidelines in Oncology. Colon Cancer, Version 1, 2012. https://www.nccn.org/professionals/physician_gls/f_guidelines.asp. Accessed August 30, 2011.

9. Fong Y and Bentrem DJ: CASH (chemotherapy-associated steatohepatitis) costs. Ann Surg 243: 8-9, 2006.

10. Maor $\mathrm{Y}$ and Malnick S: Liver injury induced by anticancer chemotherapy and radiation therapy. Int J Hepatol 2013: 815105, 2013.

11. Pawlik TM, Olino K, Gleisner AL, Torbenson M, Schulick R and Choti MA: Preoperative chemotherapy for colorectal liver metastases: Impact on hepatic histology and postoperative outcome. J Gastrointest Surg 11: 860-868, 2007.

12. Vauthey JN, Pawlik TM, Ribero D, Wu TT, Zorzi D, Hoff PM, Xiong HQ, Eng C, Lauwers GY, Mino-Kenudson M, et al: Chemotherapy regimen predicts steatohepatitis and an increase in 90-day mortality after surgery for hepatic colorectal metastases. J Clin Oncol 24: 2065-2072, 2006.

13. Tamandl D, Klinger M, Eipeldauer S, Herberger B, Kaczirek K, Gruenberger B and Gruenberger T: Sinusoidal obstruction syndrome impairs long-term outcome of colorectal liver metastases treated with resection after neoadjuvant chemotherapy. Ann Surg Oncol 18: 421-430, 2011.

14. Laurent A, Nicco C, Tran Van Nhieu J, Borderie D, Chéreau C, Conti F, Jaffray P, Soubrane O, Calmus Y, Weill B and Batteux F: Pivotal role of superoxide anion and beneficial effect of antioxidant molecules in murine steatohepatitis. Hepatology 39 : 1277-1285, 2004.
15. Pessayre D, Berson A, Fromenty B and Mansouri A: Mitochondria in steatohepatitis. Semin Liver Dis 21: 57-69, 2001.

16. Rubbia-Brandt L, Audard V, Sartoretti P, Roth AD, Brezault C, Le Charpentier M, Dousset B, Morel P, Soubrane O, Chaussade S, et al: Severe hepatic sinusoidal obstruction associated with oxaliplatin-based chemotherapy in patients with metastatic colorectal cancer. Ann Oncol 15: 460-466, 2004.

17. Nakano H, Oussoultzoglou E, Rosso E, Casnedi S, Chenard-Neu MP, Dufour P, Bachellier P and Jaeck D: Sinusoidal injury increases morbidity after major hepatectomy in patients with colorectal liver metastases receiving preoperative chemotherapy. Ann Surg 247: 118-124, 2008.

18. Dyson JK, McPherson S and Anstee QM: Non-alcoholic fatty liver disease: Non-invasive investigation and risk stratification. J Clin Pathol 66: 1033-1045, 2013.

19. Ohsawa I, Ishikawa M, Takahashi K, Watanabe M, Nishimaki K, Yamagata K, Katsura K, Katayama Y, Asoh S and Ohta S: Hydrogen acts as a therapeutic antioxidant by selectively reducing cytotoxic oxygen radicals. Nat Med 13: 688-694, 2007.

20. Hayashida K, Sano M, Ohsawa I, Shinmura K, Tamaki K, Kimura K, Endo J, Katayama T, Kawamura A, Kohsaka S, et al: Inhalation of hydrogen gas reduces infarct size in the rat model of myocardial ischemia-reperfusion injury. Biochem Biophys Res Commun 373: 30-35, 2008

21. Buchholz BM, Kaczorowski DJ, Sugimoto R, Yang R, Wang Y, Billiar TR, McCurry KR, Bauer AJ and Nakao A: Hydrogen inhalation ameliorates oxidative stress in transplantation induced intestinal graft injury. Am J Transplant 8: 2015-2024, 2008.

22. Sun Q, Kang Z, Cai J, Liu W, Liu Y, Zhang JH, Denoble PJ, Tao $\mathrm{H}$ and Sun $\mathrm{X}$ : Hydrogen-rich saline protects myocardium against ischemia/reperfusion injury in rats. Exp Biol Med (Maywood) 234: 1212-1219, 2009.

23. Fukuda K, Asoh S, Ishikawa M, Yamamoto Y, Ohsawa I and Ohta SP: Inhalation of hydrogen gas suppresses hepatic injury caused by ischemia/reperfusion through reducing oxidative stress. Biochem Biophys Res Commun 361: 670-674, 2007.

24. Cai J, Kang Z, Liu WW, Luo X, Qiang S, Zhang JH, Ohta S, Sun $\mathrm{X}, \mathrm{Xu} \mathrm{W}$, Tao $\mathrm{H}$ and Li R: Hydrogen therapy reduces apoptosis in neonatal hypoxia-ischemia rat model. Neurosci Lett 441: 167-172, 2008

25. Fu Y, Ito M, Fujita Y, Ito M, Ichihara M, Masuda A, Suzuki Y, Maesawa S, Kajita Y, Hirayama M, et al: Molecular hydrogen is protective against 6-hydroxydopamine-induced nigrostriatal degeneration in a rat model of Parkinson's disease. Neurosci Lett 453: 81-85, 2009.

26. Nakashima-Kamimura N, Mori T, Ohsawa I, Asoh S and Ohta S: Molecular hydrogen alleviates nephrotoxicity induced by an anti-cancer drug cisplatin without compromising anti-tumor activity in mice. Cancer Chemother Pharmacol 64: 753-761, 2009.

27. Liu Y, Yang L, Tao K, Vizcaychipi MP, Lloyd DM, Sun X, Irwin MG, Ma D and Yu W: Protective effects of hydrogen enriched saline on liver ischemia reperfusion injury by reducing oxidative stress and HMGB1 release. BMC Gastroenterol 14: 12, 2014.

28. Liu Q, Shen WF, Sun HY, Fan DF, Nakao A, Cai JM, Yan G, Zhou WP, Shen RX, Yang JM and Sun XJ: Hydrogen-rich saline protects against liver injury in rats with obstructive jaundice. Liver Int 30: 958-968, 2010.

29. Xu XF and Zhang J: Saturated hydrogen saline attenuates endotoxin-induced acute liver dysfunction in rats. Physiol Res 62: 395-403, 2013.

30. Levitt MD and Bond JH Jr: Volume, composition, and source of intestinal gas. Gastroenterology 59: 921-929, 1970. 